Ann. Zootech., I972, 21 (4), 567-574.

\title{
ÉVOLUTION DE LA LACTACIDÉMIE AU COURS D'UNE EXCITATION ÉLECTRIQUE CHEZ LE PORC. RELATIONS AVEC CERTAINES CARACTÉRISTIQUES PHYSICO- CHIMIQUES DU TISSU MUSCULAIRE POST MORTEM
}

\author{
G. MONIN \\ avec la collaboration technique de A. Talmant et P. Vernin \\ Station de Recherches sur la Viande, \\ Centre de Recherches de Clermont-Ferrand, I. N.R.A., \\ Saint Genès Champanelle \\ 63110 Beaumont
}

RÉSUMÉ

L.es variations de la lactacidémie au cours d'une excitation, ont été étudiées chez le Porc au moyen du dosage en continu, in vivo, de l'acide lactique sanguin. L'évolution de cette caractéristique est très variable selon les individus, et n'est pas liée aux taux de pigments transporteurs d'oxygène du muscle et du sang. Aucune relation étroite n'est établie entre l'intensité ou la durée de la réaction à l'excitation et les caractéristiques physico-chimiques du tissu musculaire, mesurées 24 heures après l'abattage.

\section{INTRODUCTION}

Il est établi depuis de nombreuses années que les excitations diverses subies avant l'abattage sont susceptibles de modifier certaines caractéristiques de la viande de porc, telles que couleur, $\mathrm{pH}$ et rétention d'eau (GOUTEFONGEA, I963). On ne sait pas toutefois avec certitude si l'intensité de ces modifications est liée à des différences individuelles de sensibilité des animaux.

Dans un but de sélection, plusieurs auteurs ont, au cours de ces dernières années, essayé de mesurer cette sensibilité par l'étude de la réaction à divers types d'agressions (STeinhauf et al., ig69; Charpentrier, I970 ; HaAse et St'teinhauf, I97 I CharpenTIER et al., I97I), mais peu de résultats susceptibles d'application pratique ont été rapportés. 
On sait que la lactacidémie est largement modifiée par la sécrétion d'adrénaline et les contractions musculaires qui accompagnent inévitablement l'excitation. D'autre part LUDVIGSEN (I957) rapporte que chez des animaux donnant post mortem des viandes à caractère exsudatif très prononcé, l'élévation du taux d'acide lactique sanguin est, à l'issue d'un effort physique, beaucoup moins intense que chez des animaux normaux; il attribue ce phénomène à une séquestration de l'acide lactique dans le muscle. I1 nous a semblé que la mise en œuvre d'une technique de dosage en continu in vivo, permettant de suivre à tout instant, et de façon sûre, les variations de la lactacidémie, pourrait nous aider à préciser les relations éventuelles entre la sensibilité des porcs à l'excitation, le transfert de l'acide lactique du muscle dans le sang et les caractéristiques ultimes du tissu musculaire post mortem.

\section{MATÉRIEL ET MÉTHODES}

42 porcs, dont 26 de race Large White et I6 de race Piétrain constituaient le matériel expérimental utilisé pour cette étude. Dans chaque race, la moitié des animaux était utilisée pour les mesures physiologiques. L'autre moitié servait de témoin, afin de déceler une influence éventuelle des manipulations nécessitées par les mesures in vivo sur la qualité des viandes obtenues après abattage. Les porcs Large White pesaient i to $\pm 5 \mathrm{~kg}$, les porcs Piétrain $95 \pm 5 \mathrm{~kg}$.

Les animaux destinés aux mesures in vivo étaient anesthésiés par injection de $\mathrm{I} g$ de thiopental sodique (Pentothal) par voie intraveineuse, la narcose était ensuite maintenue pendant le temps nécessaire (environ une demi-heure) grâce à l'administration de pentobarbital sodique (Nembutal). Un cathéther (diamètre extérieur : $1,2 \mathrm{~mm}$; longueur : $60 \mathrm{~cm}$ ) était alors introduit dans la veine médiane ou marginale postéricure de l'oreille et enfoncé d'environ $40 \mathrm{~cm}$, de façon que son extrémité se trouve dans la veine jugulaire. Cette opération était réalisée selon la technique préconisée par ANDERSON et ElsLey (I969) légèrement modifiée. Les porcs étaient alors placés dans une cage individuelle, nourris à volonté, et recevaient quotidiennement par voie orale $30 \mathrm{mg}$ d'un composé anticoagulant dicoumarinique (Adoisine).

3 à 4 jours après l'opération, un prélèvement de sang était effectué pour le dosage de l'hémoglobine (1). Puis, après injection de ro ooo UI d'héparine, le cathéter était relié à un autoanalyseur, et l'acide lactique sanguin déterminé de façon continue, après dialyse à $37^{\circ}$ pendant environ deux minutes contre de l'eau physiologique, par la méthode de HoHorst (1963) adaptée à l'autoanalyseur. Le débit du prélèvement sanguin était de $0,4 \mathrm{ml}$ par minute.

Une demi-heure environ après le début du dosage, les animaux recevaient 6 décharges électriques, espacées de 5 secondes, à l'aide d'un bâton électrique de 4,5 volts (type Villette); la détermination de la lactacidémie était poursuivie pendant environ 2 heures après cette excitation.

3 jours plus tard, après une période de jeûne de 24 heures, Ies animaux étaient abattus de façon classique, sous électronarcose, en même temps qu'un nombre égal de témoins, n'ayant subi aucune manipulation préalable.

Aussitôt après la saignée, un échantillon était prélevé et le $\mathrm{pH}$ mesuré sur le muscle L.ongissimus dorsi, au niveau des premières vertèbres lombaires. Ces opérations étaient répétées $\mathbf{1}, 2$ et 4 heures plus tard. Les mesures de $\mathrm{pH}$ étaient effectuées également sur les animaux témoins.

Les mesures de $\mathrm{pH}$ étaient réalisées à l'aide d'un $\mathrm{pH}$-mètre portatif EIL $30 \mathrm{C}$,directement sur la coupe fraîche du muscle.

Les échantillons prélevés étaient aussitôt plongés dans l'azote liquide, puis extraits dans l'acide perchlorique $0,6 \mathrm{~N}$ selon la technique décrite par CHARPENTIER (1968); l'acide lactique était ensuite déterminé sur les extraits par la méthode de HoHorst (I963) adaptée à l'autoanalyseur.

Après la dernière mesure de $\mathrm{pH}$, c'est-à-dire quatre heures post mortem toutes les carcasses étaient placées à $+6^{\circ} \mathrm{C} .24$ heures plus tard, des échantillons étaient prélevés sur les muscles Longissimus dorsi, Gluteus medius, et Biceps femoris et les caractéristiques suivantes déterminées :

- $\mathrm{pH}$ à l'aide d'un $\mathrm{pH}$-mètre EIL $30 \mathrm{C}$,

- pourcentage d'eau perdue par compression par la méthode de Goutefongea (I966),

- intensité de la coloration, mesurée par le pourcentage de rémission à 525 mu obtenu à l'aide d'un réflectomètre de CHARPENTIER-VERGÉ,

- quantité de pigment par la méthode de Hornsey (I956).

(t) Hemopack. Ets Biotrol, Paris. 


\section{RÉSULTATS ET DISCUSSION}

\section{I. - Évolution de la lactacidémie au cours de l'excitation}

Les taux d'acide lactique sanguins au repos que nous observons dans le tableau $\mathbf{r}$ sont comparables à ceux que rapportent d'autres auteurs (HAaSE et STEINHAUf, I97I) ; le niveau de la lactacidémie, légèrement supérieur chez le Large White, s'explique peut-être par le fait que ces animaux paraissent en dehors de toute perturbation extérieure, généralement plus agités que les Piétrain.

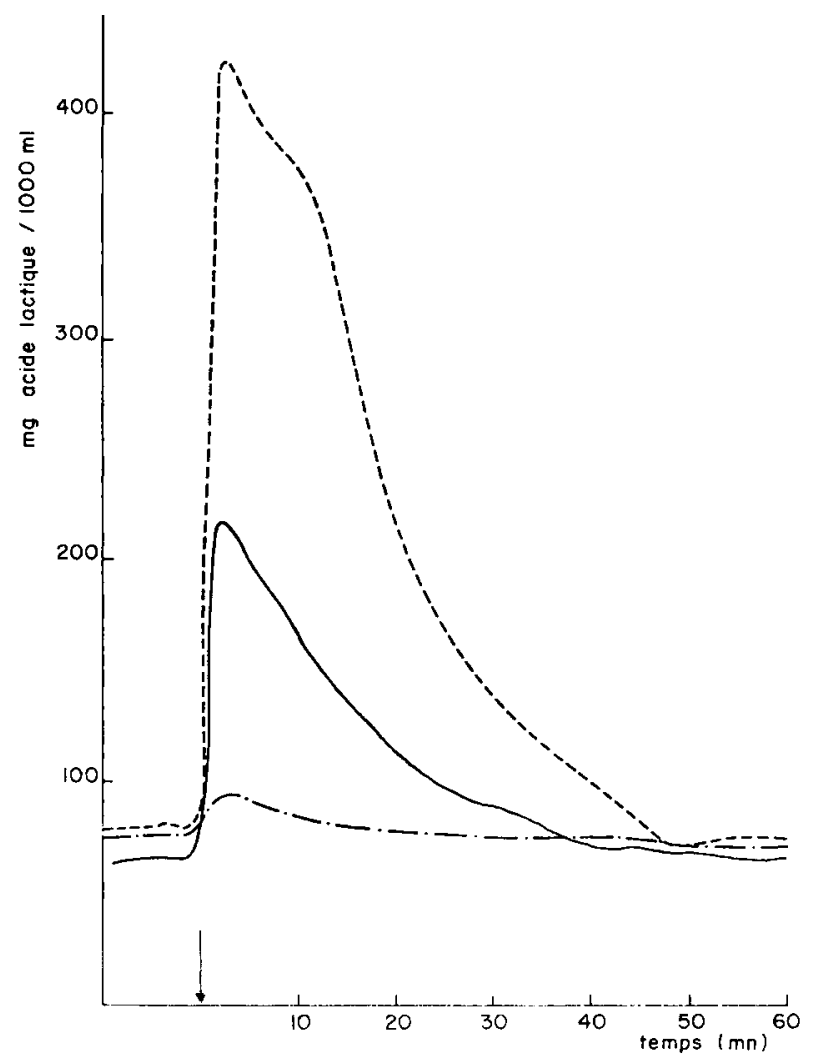

FIG. I. - Exemples de courbes individuelles d'évolution de la laclacidémie après excitation ches le Pırc

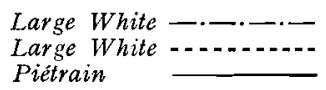

L'étude des courbes d'évolution de la lactacidémie (fig. I) montre que la valeur maximale du taux d'acide lactique sanguin est atteinte immédiatement après l'excitation ; on assiste ensuite à une diminution progressive aboutissant finalement à une valeur identique à celle que l'on observait avant l'excitation. En moyenne, chez le Piétrain, la lactacidémie atteint un niveau plus élevé et le retour à la normale est 
plus lent (tab1. I). (L'indication " durée de la réaction" mesure le temps écoulé entre l'excitation et le retour à la lactacidémie de repos). Toutefois les variations individuelles sont dans chaque race très supérieures à cette différence raciale (fig. I). La variabilité très élevée observée dans la race Piétrain pour la lactacidémie maximale (tabl. I) est due à la très haute valeur atteinte par un individu (84I mg/I $000 \mathrm{ml}$ ).

TABLEAU I

Évolution de la lactacidémie au cours d'une excitation chez le Porc

\begin{tabular}{|c|c|c|}
\hline & Large White & Piêtrain \\
\hline Lactacidémie au repos mg/l & $90,6 \pm 15,3^{*}$ & $68,4 \pm 16,6^{*}$ \\
\hline Lactacidémie après excitation $\mathrm{mg} / 1$. & $271,7 \pm 143,6$ & $280,9 \pm 250,7$ \\
\hline Durée de la modification (mn) ..... & $34,5 \pm 10,4$ & $41,4 \pm 10,3$ \\
\hline
\end{tabular}

* Différence significative au seuil de probabilité de 5 p. 100.

La lactacidémie est à tout moment la résultante de la production d'acide lactique dans certains organes, principalement le muscle, et de sa captation par d'autres organes, en tout premier lieu le foie (CORI et CORI, I928). L'excitation doit donc refléter à la fois 1'intensité de la glycogénolyse musculaire anaérobie et le passage de l'acide lactique dans le sang, tandis que la phase de retour traduit la fixation hépatique de l'acide. Des variations de la fonction hépatique expliquent sans doute pour une part les différences individuelles importantes dans le délai de retour à la lactacidémie normale, puisque la corrélation entre ce délai et l'intensité de la réaction à l'excitation (mesurée par la lactacidémie maximale) est, quoique significative relativement faible $(r=0,52$; tabl. 2$)$.

\section{TABLEAU 2}

Corvélations simples entre divers paramètres physiologiques

\begin{tabular}{|c|c|c|c|c|c|c|}
\hline & $\begin{array}{c}\text { Lactacidémie } \\
\text { maximale }\end{array}$ & $\begin{array}{l}\text { Durée de la } \\
\text { modification }\end{array}$ & Hémoglobine & $\begin{array}{l}\text { Pigment } \\
\text { LD (1) }\end{array}$ & $\begin{array}{l}\text { Pigment } \\
\text { GM (1) }\end{array}$ & $\begin{array}{l}\text { Pigment } \\
\text { BF (1) }\end{array}$ \\
\hline Lactacidémie repos $\ldots .$. & 0,12 & $-0,31$ & $-0,31$ & 0,11 & 0,30 & 0,41 \\
\hline Lactacidémie maximale .. & & $0,52 *$ & $-0,28$ & $-1,12$ & $-0,15$ & $-0,19$ \\
\hline Durée de la modification & & & 一 $0,4: 3$ & 0,10 & 0,11 & 0,09 \\
\hline
\end{tabular}

* Corrélation significative au seuil de probabilité de 5 p. 100.

(1) Abréviations utilisées $\left\{\begin{array}{l}\mathrm{LD}: \text { Longissimus dorsi } \\ \mathrm{GM}: \text { Gluteus medius } \\ \mathrm{BF}: \text { Biceps femoris }\end{array}\right.$ 
Il est connu que la glycolyse musculaire est accélérée lorsque l'apport d'oxygène au muscle devient insuffisant (MORGAN et PARMEGGIANI, I964). I1 est possible que, au cours de la situation coûteuse en énergie que représente la réaction à l'excitation, la "dette d'oxygène " au niveau du muscle présente une intensité variable selon les individus. Les résultats du tableau 2 montrent que ce phénomène n'est pas lié à une déficience en hémoglobine ou myoglobine, les deux pigments transporteurs d'oxygène quantitativement les plus importants dans l'organisme : en effet les corrélations entre les taux de pigments sanguins ou musculaires et les variations de la lactacidémie sont très faibles. Toutefois, l'approvisionnement en oxygène des cellules musculaires peut être perturbé par des modifications de la microcirculation sanguine (LISTER et al., I970) ; d'autre part l'efficacité de l'utilisation de l'oxygène dans les mitochondries est sujette chez le porc à des variations importantes selon les races et les individus (EIKEIENBOOM et VAN DEN BERGH, I97I). D'après ces auteurs, elle est particulièrement faible chez les Piétrain, qui montrent ici les taux d'acide lactique sanguin les plus élevés après l'excitation.

\section{2. - Relations entre la réaction à l'excitation}

et quelques caractéristiques physico-chimiques du muscle post mortem

Les résultats du tableau 3 montrent que les traitements subis par les animaux expérimentaux ont eu peu de conséquences sur la glycogénolyse musculaire post mortem et les caractéristiques de la viande. Nous pouvons donc considérer, comme

\section{TABLEAU 3}

Chute du pH musculaive et caractéristiques de qualité de la viande : influence des traitements et différences raciales

\begin{tabular}{|c|c|c|c|c|c|}
\hline & & \multicolumn{2}{|c|}{ Traitement } & \multicolumn{2}{|c|}{ Race } \\
\hline & & $\begin{array}{l}\text { Expéri- } \\
\text { mentaux }\end{array}$ & Témoins & Large White & Piétrain \\
\hline \multirow{5}{*}{$\mathrm{pH}$ Longissimus dorsi } & $0 \mathrm{~h} p . m$. & $6,65+0,24$ & $6,74 \pm 0,24$ & $6,63+0,20$ & $6,68+0,16$ \\
\hline & $1 \mathrm{~h} \mathrm{p.m.}$ & $6,12 \pm 0,42$ & $6,33 \pm 0,34$ & $6,23 \pm 0,40$ & $5,92 \pm 0,39$ \\
\hline & $2 \mathrm{~h} p . m$ & $5,91 \pm 0,32$ & $6,06 \pm 0,33$ & $5,98 \pm 0,34$ & $5,75 \pm 0,23$ \\
\hline & $4 \mathrm{~h} p . m$ & $5,65 \pm 0,25$ & $5,77 \pm 0,29$ & $5,70 \pm 0,28$ & $5,54 \pm 0,11$ \\
\hline & $0 \mathrm{~h} p . m$. & & & $29,6 \pm 12,1$ & $37,7 \pm 11,3$ \\
\hline \multirow{4}{*}{$\begin{array}{c}\text { Acide lactique LD } \\
(\mu \mathrm{M} / \mathrm{g} \text { muscle })\end{array}$} & $1 \mathrm{~h} p . m$ & & & $44,7 \pm 18,4^{*}$ & $67,1 \pm 20,3^{*}$ \\
\hline & $2 \mathrm{~h} \mathrm{p.m.}$ & & & $68,9 \pm 24,1^{*}$ & $98,1 \pm 20,9^{*}$ \\
\hline & $4 \mathrm{~h} p . m$. & & & $78,9 \pm 21,3^{*}$ & $98,7 \pm 11,7^{*}$ \\
\hline & Longissimus dorsi & $5,55 \pm 0,12$ & $5,55 \pm 0,11$ & $5,53 \pm 0,1_{t}^{\prime}$ & $5,54 \pm 0,10$ \\
\hline \multirow[t]{2}{*}{$\mathrm{pH}, 24 \mathrm{H}$ p.m. } & Gluteus medius & $5,55 \pm 0,12$ & $5,56 \pm 0,11$ & 5,53 上 0,15 & $5,58 \pm 0,07$ \\
\hline & Biceps femoris & $5,57 \pm 0,12$ & $5,57 \pm 0,12$ & $5,57 \pm 0,14$ & $5,59 \pm 0,09$ \\
\hline \multirow{3}{*}{ P. 100 eau libre } & Longissimus dorsi & $27,7 \pm 4,4$ & $27,6 \pm 3,1$ & $26,5 \pm 3,5$ & $28,8 \pm 5,6$ \\
\hline & Gluteus medius & $28,7 \pm 4,0$ & $28,5 \pm 3,2$ & $27,8 \pm 4,0$ & $30,2 \pm 3,6$ \\
\hline & Biceps femoris & $28,2 \pm 3,0$ & $27,3 \pm 5,0$ & $27,1 \pm 3,4$ & $26,7 \pm 1,9$ \\
\hline & Longissimus dorsi & $861 \pm 107$ & $797 \pm 107$ & $803 \pm 85^{* *}$ & $956 \pm 66^{* *}$ \\
\hline \multirow[t]{2}{*}{ Réflectance \{} & Gluteus medius & $81^{\prime} \pm 128$ & $781 \pm 128$ & $767 \pm 115^{*}$ & $891 \pm 115^{*}$ \\
\hline & Biceps femoris & $765 \pm 107$ & $763 \pm 111$ & $752 \pm 107$ & $795 \pm 93$ \\
\hline
\end{tabular}

* Différence significative au seuil de probabilité de 5 p. 100.

** - $\quad$ - $\quad$ - $\quad$ - $\quad$ de 1 p. 100. 
G. MONIN

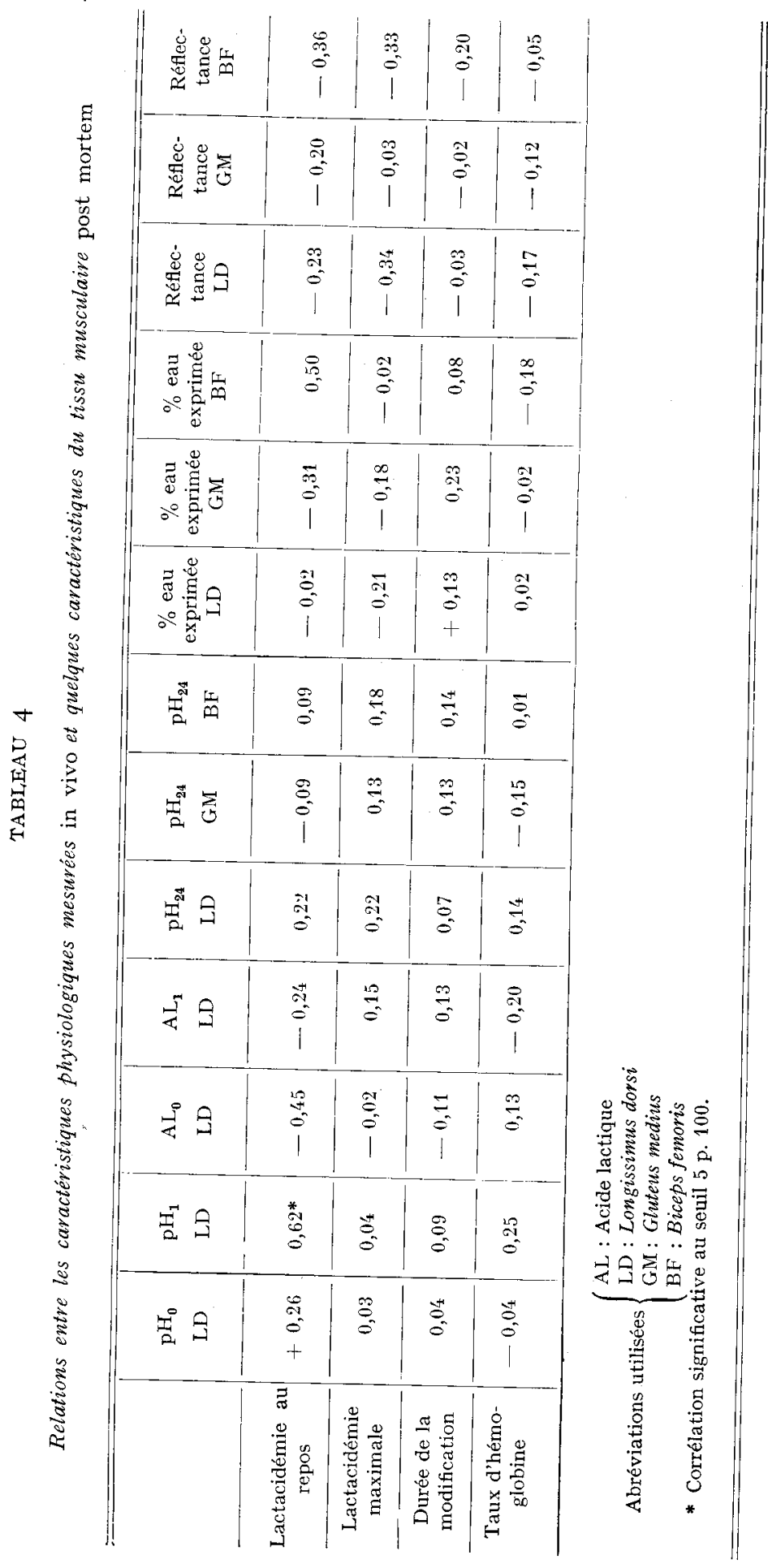


normaux du point de vue de ces différentes caractéristiques, les porcs ayant servi aux mesures in vivo.

En moyenne, les animaux de race Piétrain, qui présentent une viande de qualité légèrement inférieure à celle des Large White, réagissent par une augmentation de la lactacidémie plus prononcée $(+2 \mathrm{I} 2 \mathrm{mg} /$ litre contre $+\mathrm{I} 80 \mathrm{mg}$ pour les Large White). Toutefois les corrélations calculées sur l'ensemble de l'effectif entre les résultats des mesures in vivo et les caractéristiques de qualité de la viande sont pour la plupart très faibles (tabl. 4). En outre, une éventuelle séquestration de l'acide lactique dans le muscle devrait se traduire, soit par un retard de l'apparition de l'acide dans le sang, soit par une élévation moindre de la lactacidémie, comme le rapporte LUDVIGSEN (I957); or chez tous les animaux, quelles que soient les caractéristiques du tissu musculaire observées post mortem, l'augmentation du taux d'acide lactique sanguin succède immédiatement à 1'excitation. Nos résultats ne confirment donc pas ceux de LUDVIGSEN, obtentus, il est vrai sur des pores atteints de " dégénérescence musculaire " (" muscular degeneration ") héréditaire, véritable état pathologique, donc probablement très éloignés physiologiquement des porcs réputés normaux que nous avons utilisés.

En définitive, nos observations confirment les travaux de Steinhauf et al. (I969), Charpentier et al. (I97I), HaAse et Steinhauf (I97I), selon lesquels les modalités de la réaction à une agression isolée ne sont pas liées directement aux perturbations du métabolisme musculaire qui conduisent à l'obtention post mortem de viandes exsudatives. En particulier, la réaction à l'excitation met en jeu des mécanismes qui n'ont que peu d'influence sur la vitesse de glycogénolyse post mortem, par exemple la sécrétion d'adrénaline (Charpentíer et Monin, résultats non publiés)

\section{CONCLUSTON}

Le porc manifeste une sensibilité généralement importante, mais très variable avec les individus, à des excitations légères comme l'application de décharges électriques modérées. Au cours de telles excitations la lactacidémie augmente très rapidement, mais de façon transitoire, et le retour à la normale est relativement rapide.

De nombreux travaux ont montré qu'une granđe sensibilité à divers types d'agressions (chaleur, contention, exercice forcé...) caractérise les races qui donnent habituellement des viandes de qualité défectueuse. En France, ce fait est bien connu pour la race Piétrain. Toutefois, nous n'avons pu établir, sur la base de critères simples comme les modifications de la lactacidémie ou d'autres caractéristiques physiologiques (Charpentier et al., I97I), l'existence de relations directes entre l'intensité de la réaction à une agression et les perturbations métaboliques responsables de 1'apparition du caractère exsudatif dans la viande. Il est probable que la multiplicité des mécanismes de défense mis en jeu lors de l'agression masque l'effet propre de telles perturbations. Aussi, apparaît-il indispensable, au cours d'expériences ultérieures, de préciser quels sont parmi ces mécanismes ceux qui sont susceptibles d'avoir une influence directe sur le déroulement de la glycogénolyse musculaire post mortem. 


\section{SUMMARY}

\section{CHANGES IN BLOOD LACTIC ACID LEVEL DURING EXCITEMENT}

\section{AND MEAT QUALITY IN PIGS}

The effect of excitement on the blood lactic acid level was studied in I 3 Large White pigs and 8 Pietrain pigs. A catheter was inserted in the jugular vein of each pig according to ANDERSON and ELSLEY (I969), and blood lactic acid was determined in vivo owing to an Autoanalyzer. Application of discharges from an electric rod results in a sharp rise of blood lactic acid. Large individual differences among animals were recorded.

After slaughtering, $\mathrm{pH}$ fall was observed and meat quality measured owing to $\mathrm{pH}$, colour and waterbinding capacity measurements. The experimental animals were compared to an equal number of control of each breed, but no influence of experimental treatment was found. No relationship between susceptibility to excitement and meat quality characteristics was observed.

\section{RÉFÉRENCES BIBLIOGRAPHIQUES}

Anderson D. M., Elsley F. W. H., I969. A note on the use of indwelling catheters in conscious adult pigs. J. Agric. Sci., 72, 475-478.

Charpentier J., 1968. Glycogénolyse post mortem du muscle Longissimus dorsi de Porc. Ann. Zootech., 17, 429-443.

- Charpentier J., i97o. Relation entre la réaction physiologique du porc à un stress thermique et le caractere exsudatif du tissu musculaire post mortem XVIth European meeting of meat research workers. Varna, Tome I, 57-68.

Charpentier J., Monin G., Oldivier L., r97I. Relations entre les réactions du porc à un choc thermique et la qualité de la viande. Journées études Commiss. Génét. F.E.Z. Versailles, I7-19 juillet I97I.

Cori C. J., Cori G. T., 1928. Epinephrine in fasting rats. J. Biol. Chem., 79, 275.

Eikelenboom C., VAN DeN BeRGH S. G., I97I. Aberrant mitochondrial energy metabolism in stress susceptible pigs. in Proceedings of the Second Symp. on Conditions and Meat Quality of pigs, Zeist, March 22-24, I97I. Ed. Centre Agr. Publ. Doc. Wageningen. The Netherlands.

GoutefongeA R., 1963. Les viandes exsudatives. Ann. Zootech., 12, 297-337.

Goutefongea R., ig66. Etude comparative de différentes méthodes de mesure du pouvoir de rétention d'eau de la viande de Porc. Ann. Zootech., 15, 29 I-295.

HaAse S., Steinhauf D., I97r. Effects of stress on some oxygen metabolism parameters in boars, in Proceedings of the Second Symp. on Conditions and Meat Quality of pigs, Zeist, march 22-24, 1971. Ed. Centre Agr. Publ. Doc. Wageningen. The Netherlands.

Hohorst H. S., 1963. L $(+)$ Lactate. Determination with Lactic Dehydrogenase and DPN, in BERGMEYer H.-V. Methods of enzymatic analysis 266-27o. Acad Press, New York.

HoRRSEY H.-C., I956. The colour of cooked cured pork. I. Estimation of the nitric-oxide-haem pigments. J. Sci. Food Agric., 7, 534-540.

Lister D., Sair R. A., Will J. A., Schmidt G. R., Cassens R. G., Hoekstra W. G., Briskey E. J., I970. Metabolism of striated muscle of stress-susceptible pigs breathing oxygen or nitrogen. Am. J. Physiol., 218, т02-I07.

LUdVigsen J., I957. On the hormonal regulation of vasomotor reactions during exercise with special reference to the action of adrenal cortical steroids. Acta Endocrinologica, 76, 406-4I6.

Morgan H. E., Parmeggiani A., 1964. Regulation of glycolysis in muscle. In Control of glycogen metabolism. Ed. J. A. Churchill. Ltd London.

Steinhauf D., Weniger J. H., Hoppenbrock K. H., I969. Stressresistenz als Leistungsmerkmal beim Schwein. III. Mitteilung: Weitere Untersuchungen über Beziehungen zwischen Merkmalen der Stressanfälligkeit und leistungseigenschaften beim Schwein. Zugleich eine kritische Betrachtung zur Theorie des allgemeinen Anpassungssyndrom. Züchtungskunde, 41, 93-1 I I. 